\title{
NEW DIELECTRIC - DOUBLE TETRAMETAPHOSPHATE $\mathrm{Li}_{2} \mathrm{Mn}\left(\mathrm{PO}_{3}\right)_{4}$
}

\author{
R. V. LAVRYK, PhD in Chemistry \\ D. M. KAMYSHAN, student \\ V. V. POLISHCUK, student \\ National University of Life and Environmental Sciences of Ukraine
}

Alkaline metal fluorides influence on the solubility of oxides of manganese, nickel and cobalt in fluorophosphate systems of alkaline metals has been investigated. It was found that the solubility of the corresponding oxides of $3 \mathrm{~d}$-metals grows from lithiumto potassium fluorophosphate systems. Sites of formation, composition and properties of the obtained double phosphates were established using physico-chemical methods. The main factors influencing the composition of the phosphate compounds were identified. Specific features of crystal structure of double phosphate were studied. The optimum conditions of monocrystal growth were suggested.

Introduction. The progress of inorganic chemistry is syntheses of new phosphates. We learned of structure of new double phosphate $\mathrm{Li}_{2} \mathrm{Mn}\left(\mathrm{PO}_{3}\right)_{4}$ by RSA methods. This phosphate is obtained from lows of systems $\mathrm{Li}_{2} \mathrm{O}-\mathrm{P}_{2} \mathrm{O}_{5}$.

RSA of white crystals ortorombicgabitus with parametrs $0,2 \times 0,019 \times 0,2 \mathrm{~mm}$ study on difractometr "Siemens P3/PC" in carbon monocromator. Structure of $\mathrm{Li}_{2} \mathrm{Mn}\left(\mathrm{PO}_{3}\right)_{4}$ is ortor. syng.,Pnma, parametrs of frame: $\mathrm{a}=9,268(1), \quad \mathrm{b}=9,421(1), \quad \mathrm{c}=10,088(1) \quad \mathrm{A}$, $\mathrm{V}=880,9 \mathrm{~A}^{3}, \mathrm{Z}=4$, $\rho$ cal $=2,901 \mathrm{~g} / \mathrm{sm}^{3}$.

Powder diffraction data for structure determination were obtained at room temperature in Bragg-Brentano [1]geometry using a Siemens D500 powder diffractometer equipped with a primary $\mathrm{SiO}_{2}$ monochromator ( $\mathrm{Cu} \mathrm{Ka} \mathrm{Ka}_{1}$ radiation, $\mathrm{X} .=1.54060 \AA$ ) and $\mathrm{a}$ position sensitive detector (Braun). The data were collected over the range $10-110^{\circ}$
20 with a step $\Delta(2 \theta)=0.01^{\circ}$. Effective counting time was 30 min per step.

Results and discussion. The atomic coordinates of were used as starting parameters for structure refinements. The Mncations were placed in the $M(5)$ site. Alkali metal cations

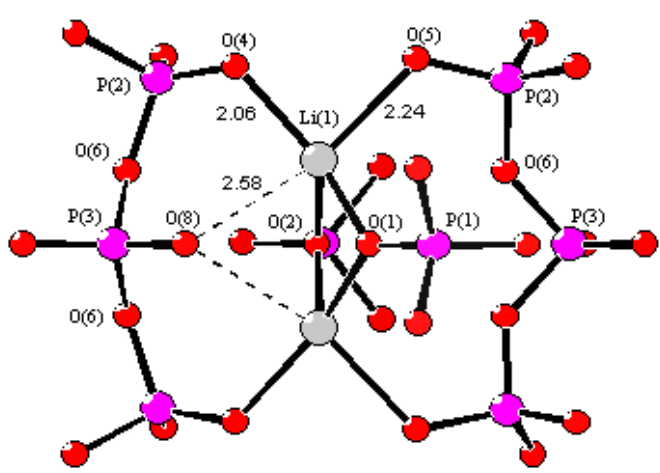

Fig. 1. Coordination of $\mathrm{Li}$-atoms in structures of $\mathrm{Li}_{2} \mathrm{Mn}\left(\mathrm{PO}_{3}\right)_{4}$.

Supervisor of studies - R.V. Lavryk 
XIMIЯ

Р. В. Лаврик, Д. М. Камишан, В. В. Поліщук

Coordination of atoms $\left(\times 10^{4}\right)$ andequivalent thermal corrections $\left(\AA^{2} \times 10^{3}\right)$ forstructure $\mathrm{Li}_{2} \mathrm{Mn}\left(\mathrm{PO}_{3}\right)_{4}$

\begin{tabular}{|c|c|c|c|c|}
\hline Atoms & $\mathbf{x} / \mathbf{a}$ & $\mathbf{y} / \mathbf{b}$ & $\mathbf{z} / \mathbf{c}$ & Ueq \\
\hline $\mathrm{Mn}(1)$ & $9876(1)$ & 7500 & $3031(1)$ & $9(1)$ \\
\hline $\mathrm{P}(1)$ & $6946(1)$ & 7500 & $6070(1)$ & $8(1)$ \\
\hline $\mathrm{P}(2)$ & $7078(1)$ & $9625(1)$ & $3896(1)$ & $8(1)$ \\
\hline $\mathrm{P}(3)$ & $7270(1)$ & 12500 & $5157(1)$ & $7(1)$ \\
\hline $\mathrm{O}(1)$ & $8501(3)$ & 7500 & $6252(3)$ & $15(1)$ \\
\hline $\mathrm{O}(2)$ & $5997(3)$ & 7500 & $7234(3)$ & $12(1)$ \\
\hline $\mathrm{O}(3)$ & $6481(2)$ & $8835(2)$ & $5177(2)$ & $20(1)$ \\
\hline $\mathrm{O}(4)$ & $8648(2)$ & $9404(2)$ & $3796(2)$ & $12(1)$ \\
\hline $\mathrm{O}(5)$ & $6166(2)$ & $9276(2)$ & $2741(2)$ & $16(1)$ \\
\hline $\mathrm{O}(6)$ & $6725(2)$ & $11236(2)$ & $4233(2)$ & $13(1)$ \\
\hline $\mathrm{O}(7)$ & $6466(3)$ & 12500 & $6397(3)$ & $18(1)$ \\
\hline $\mathrm{O}(8)$ & $8857(3)$ & 12500 & $5201(3)$ & $13(1)$ \\
\hline $\mathrm{Li}(1)$ & $9970(6)$ & $8962(7)$ & $6678(7)$ & $32(2)$ \\
\hline
\end{tabular}

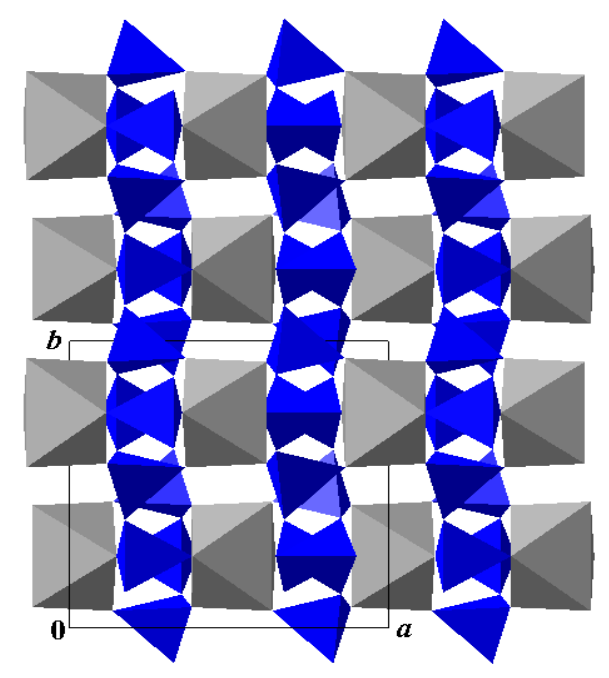

Fig. 2. Coordination of polyedres structures of $\mathrm{Li}_{2} \mathrm{Mn}\left(\mathrm{PO}_{3}\right)_{4}$. were placed in the $\mathrm{M}(4)$ site as it was obtained. The structure refinements in these models gave a good agreement between the observed and calculated patterns and reasonable values of isotropic thermal atomic parameters for all cations. Final plots of observed electron density maps did not show residual peaks [2].

The polyedrs $\left[\mathrm{MnO}_{6}\right]$ have two e日vivalent bonds $\mathrm{Mn}-\mathrm{O}(4)$ and $\mathrm{Mn}-\mathrm{O}(5)$ with long 2,261 A and 2,198 A. All atoms of oxygen of polyedres $\left[\mathrm{MnO}_{6}\right]$ have contacts with four tetraedrs $\mathrm{P}(2) \mathrm{O}_{4}$ through atoms $\mathrm{O}(5)$ and $\mathrm{O}(4)$, with tetraedrs $\mathrm{P}(1) \mathrm{O}_{4}$ and $\mathrm{P}(3) \mathrm{O}_{4}$ through atoms $\mathrm{O}(7)$ and $\mathrm{O}(8)$ (Fig. 1,2).

Interestingly, this magnetic structure was found to consist of both antiferromagnetic and ferromagnetic exchange interactions mediated through Mn-O-Mn-O pathways.

\section{References}

1. Dickens, B., Schroeder,L.W., Brown,W.E. (1994) The structures of phosphates. Journal Solid State Chemistry, 10, 232-240.

2. Sheldrick G.M. (1997) RIETAN-97. A program for crystal structure refinement. Release 97-2. University of $\mathrm{G}$ ttingend, Germany. 


\section{АНОТАЦІЯ}

Р. В. Лаврик, Д. М. Камишан, В. В. Поліиук. Новий діелектрик - подвійний тетраметафосфат $\mathrm{Li}_{2} \mathrm{Mn}\left(\mathrm{PO}_{3}\right)_{4} / /$ Біоресурси $\mathrm{i}$ природокористування. - 2016. - 8, №5-6. - C.23-26.

Стаття присвячена синтезу нового тетраметафосфату $\mathrm{Li}_{2} \mathrm{Mn}\left(\mathrm{PO}_{3}\right)_{4}$ при використанні фторидів лужних металів. За допомогофізико-хімічних методів встановлено склад та будову синтезованого фосфату, визначені його кристалографічні характеристики. Структура $\mathrm{Li}_{2} \mathrm{Mn}\left(\mathrm{PO}_{3}\right)_{4}$ належить до орторомбічної сингонї, пр. гр. Рпта, параметри кристалічної решітки дорівнюютть: $a=9,268(1), b=9,421(1), c=10,088(1) A$, $V=880,9$ A3, $Z=4, \quad \rho$ вирах. $=2,901 \mathrm{z}^{2} \mathrm{~cm}^{3}$. Поліедри [MnО $\mathrm{M}_{6}$ мають по два однакових зв'язки $\mathrm{Mn}-\mathrm{O}(4) \mathrm{ma} \mathrm{Mn-O(5)}$ довжиною 2,261 A $i$ 2,198 A. Всі атоми оксигену октаедрів [МпО6] мають контакти з чотирма тетраедрами $\mathrm{P}(2) \mathrm{O}_{4}$ через атоми $O(5) i$ $\mathrm{O}(4)$, з поліедрами $\mathrm{P}_{(1) \mathrm{O}_{4}} \mathrm{ma}^{\mathrm{P}(3) \mathrm{O}_{4}}$ через атоми $O(7)$ та $O(8)$ відповідно. Таке поєднання структурних фрагментів призводить до "стягування" октаедрів поліфосфатними ланиюжками з тетраедрів $\left[\mathrm{PO}_{4}\right]$, внаслідок чого формується жорсткий каркас структуpu $\mathrm{Li}_{2} \mathrm{Mn}\left(\mathrm{PO}_{3}\right)_{4}$.

\section{АННОТАЦИЯ}

Р. В. Лаврик, Д. М. Кампшан, В. В. Полищук. Новый диэлектрик - двойной тетраметабосфат $\mathrm{Li}_{2} \mathrm{Mn}\left(\mathrm{PO}_{3}\right)_{4} / /$ Биоресурсъь u природопользование. - 2016. - 8, №5-6. - C.23-26.

Статья посвяшена синтезу нового тетраметафосфата $\mathrm{Li}_{2} \mathrm{Mn}\left(\mathrm{PO}_{3}\right)_{4}$ при использовании фторидов шелочных металлов. С помоизю физико-химических методов установлен состав и строение синтезированного фосфата, определены его кристаллографические характеристики. Структура $\mathrm{Li}_{2} \mathrm{Mn}\left(\mathrm{PO}_{3}\right)_{4}$ принадлежит к орторомбической сингонии, пр. гр. Рпта, параметры кристаллической решетки равны: $a=9,268(1), \quad b=9,421(1)$, $c=10,088(1) A, V=880,9 A 3, Z=4, \rho в ы ч .=2,901$ г/см3. Полиэдри $\left[\mathrm{MnO}_{6}\right]$ имеют по два одинаковых связи Мn-O(4) и Мn-O(5) длиной 2,261 A и 2,198 A. Все атомъь кислорода октаэдров [МпO6] имеют контакты $c$ четьрьмя тетраэдрами $\mathrm{P}(2) \mathrm{O}_{4}$ через атомь $O(5)$ и $\mathrm{O}(4)$, с полиэдрами $\mathrm{P}(1) \mathrm{O}_{4}$ и $\mathrm{P}(3) \mathrm{O}_{4}$ через атомы $O(7)$ и (8) соответственно. Такое сочетание структурных фрагментов приводит к "стягиванию" октаэдров полифосфатными иепочками из тетраэдров [PO $]$ ], вследствие чего формируется жесткий каркас структуры $\mathrm{Li}_{2} \mathrm{Mn}\left(\mathrm{PO}_{3}\right)_{4}$. 